\title{
Performance estimation of a cell-to-cell-type active balancing circuit for lithium-ion battery systems
}

\author{
Manuel Räber, Djaffar Ould Abdeslam \\ IUT de Mulhouse, MIPS Laboratory \\ Université de Haute Alsace \\ 68093 Mulhouse Cedex, F
}

\author{
Andreas Heinzelmann, Andres Ramirez \\ Institute for Energy Systems and Fluid Engineering \\ Zurich University of Applied Sciences \\ 8400 Winterthur, $\mathrm{CH}$
}

\begin{abstract}
Active charge balancing is an approved technique to implement more energy-efficient and eco-friendly lithium-ion battery systems. The theoretical analysis presented in this paper provides a method to estimate the benefits of a cell-to-cell-type active charge balancing circuit in comparison to a passive balancing solution concerning energy savings and capacity gain. The calculation's variable parameters are the battery system configuration and the cell capacity distribution properties. Their validity is limited to applications with normally distributed cell capacities, limited maximum and minimum cell capacity and full cycle usage. The losses related to passive balancing in an $n \mathrm{SmP}$ battery system are calculated as well as the overall energy savings achievable with cell-to-cell based active balancing. The capacity gain factor of an actively balanced battery system related to a passive one is found to be in a range between 1.06 and 1.01 depending on the cell parameters and the system configuration. The derived formulas are verified by numeric simulations. Based on the results, several options are identified to increase the energy efficiency of conventional passive balancing systems. The findings can be used during the design process of new battery systems or to analyze and optimize any existing lithium-ion battery system.
\end{abstract}

Keywords - Battery management system, active charge balancing, lithium-ion battery systems, active balancing techniques

\section{INTRODUCTION}

For lithium-ion battery systems, the use of an electronic battery management system (BMS) is essential for safety and operational reasons. It prevents cell failures caused by overcharging or deep discharging, monitors the temperature, and calculates the state of charge (SoC).

Individual battery cells in a stack of multiple cells in series tend to vary in capacity, which leads to a reduced overall battery capacity as weak cells reach charge and discharge limits earlier. As long as large battery systems consisted mainly of lead-acid or $\mathrm{NiCd} / \mathrm{NiMH}$ cells uneven charge distribution was a minor issue. Overcharging these cells with the usual low charging currents would lead to a relatively harmless increase in charging losses. Lithium-ion cells, however, are much more sensitive to overvoltage. Therefore, a BMS with charge balancing function is required to prevent the battery system from damage and premature battery failure. The balancer ensures that each cell is completely charged.
Passive balancing is based on a resistive circuit to dissipate excess charge and thereby equalize the cells. Major drawbacks of passive balancing are a rather high balancing time due to the limited heat dissipation and a reduction in overall charging efficiency. Nowadays, as the demand for large lithium-ion based battery systems emerges, these drawbacks become more important to both battery system integrators and end-users.

Active balancing solutions are able to transfer charge between individual cells at high efficiency and have been discussed extensively during the past few years. They represent a promising possibility to enhance the energy efficiency and eco friendliness of lithium-ion battery systems when used both during the charging and discharging process.

In Fig. 1 the basic process is shown for both charge and discharge process. For a general topology overview see [1] and [2].

Passive Balancing

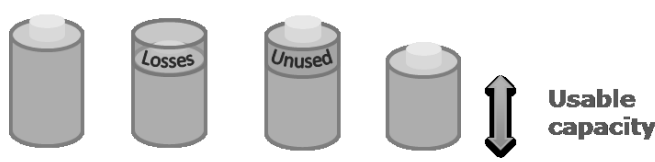

Active Balancing
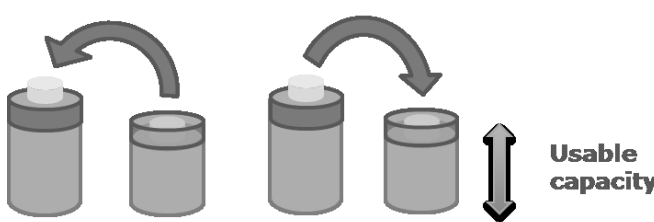

Charging

Discharging

Fig. 1. Basic function principle of active charge balancing

For information regarding hardware effort and performance comparison refer to [3], [4] or [5]. Commonly, four basic topologies are distinguished: Cell-to-cell, cell-to-stack, stackto-cell and stack-to-cell-to-stack, which is a combination of the previous two. Apart from these standard topologies other circuits and types have been proposed in [6], [7] and [8]. The main feature of active balancing is the efficient charge transfer 
from cells with high SoC to cells with low SoC. In Fig. 2 the four main topologies are shown. The cell-to-null method describes the common implementation of passive balancing through resistive dissipation.

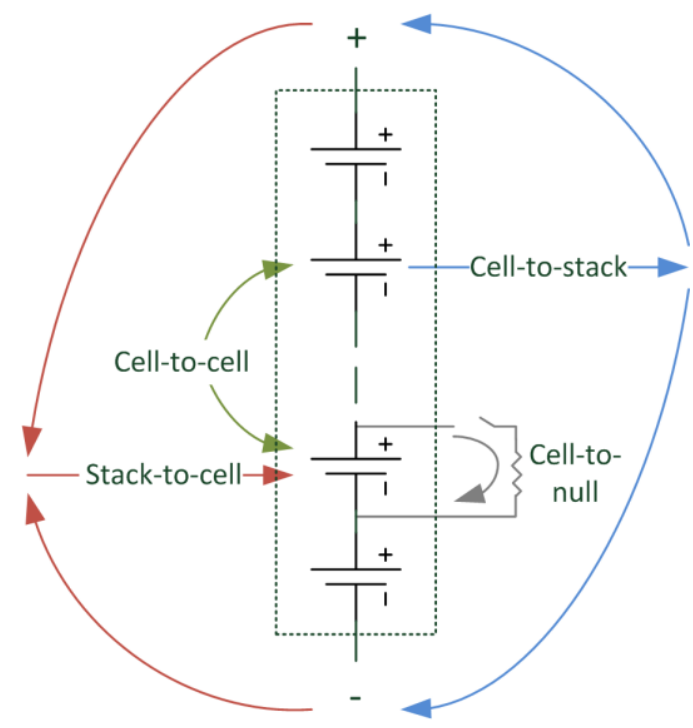

Fig. 2. Schematic overview of common charge balancing methods

In a comparison presented in [3] the cell-to-cell topology shows superior performance compared to any other active balancing methods in terms of balancing speed and efficiency. Cell-to-cell balancers are technically challenging due to the numerous power paths.

Current and hence power flow in a cell-to-cell balanced battery system can be calculated easily. Assuming identical cell voltages, the cell current vector $\vec{l}_{c e l l}$ can be expressed as a function of the stack current $I_{\text {stack }}$, the individual cell balancing currents $\vec{l}_{B a l}$, the energy efficiency of the balancing electronics $\eta$ and the balancing matrix $A$ which defines the balancing paths:

$$
\begin{gathered}
\vec{\imath}_{\text {cell }}=I_{\text {stack }}-\vec{\imath}_{\text {Bal }}+\eta \cdot A \cdot \vec{\imath}_{\text {Bal }} . \\
A=\left(\begin{array}{cccc}
0 & s_{12} & \cdots & s_{1 n} \\
\vdots & \ddots & \vdots & \vdots \\
s_{n 1} & s_{n 2} & \cdots & 0
\end{array}\right), s_{x y} \in\{0 ; 1\}
\end{gathered}
$$

The distribution of the battery cell capacities must be known to make a statement about charge imbalance in lithium-ion batteries. Measurements published in [9] support the assumption that battery cell capacities are normally distributed (see Fig. 3). Cells with a deviation of more than approximately $-2.75 \sigma$ do not show up in the histogram in Fig. 3 since they fail the factory quality test and are not shipped.

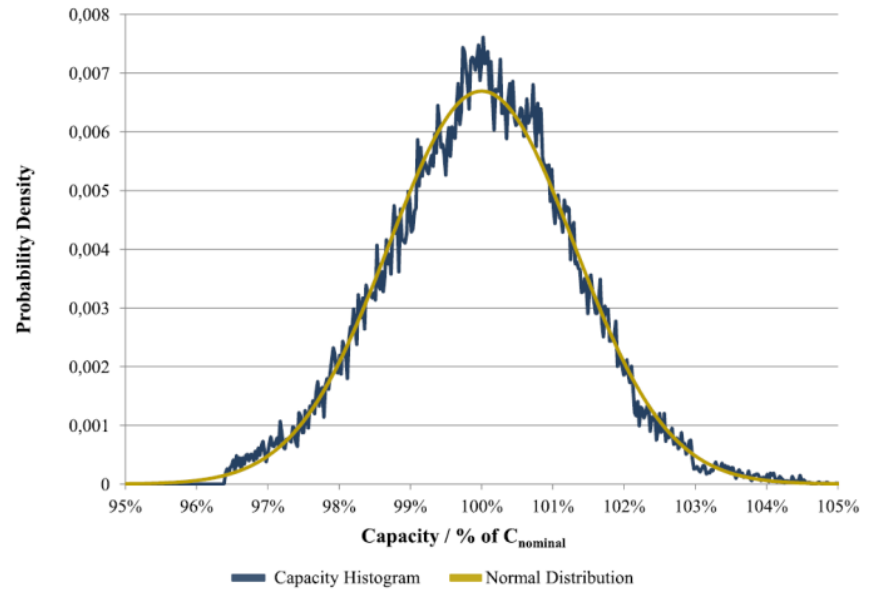

Fig. 3. Distribution of cell capacities at Beginning of Life (BoL) (20’000 measured cells, $\sigma=1.3 \%$ ) [9]

In section II, the distribution of cell capacities is described analytically. Based on the information presented in this introduction and using the new findings, formulas for the charge imbalance in a battery systems and its behavior over the lifetime are derived to allow a comparison of passive and active charge balancing.

\section{CAlculation OF THE BALANCING CHARGE}

\section{A. Capacity distribution}

We assume that the battery cell capacities are normally distributed acc. to the Gaussian density function with the variables $\mu=$ mean and $\sigma=$ standard deviation:

$$
f(x)=\frac{1}{\sqrt{2 \pi \sigma^{2}}} e^{\frac{(x-\mu)^{2}}{2 \sigma^{2}}}
$$

The mean value $E$ and the expected value $\mu$ are identical for symmetric functions:

$$
\mu=\int_{-\infty}^{+\infty} x f(x) d x
$$

The cumulative density function $F$ cannot be calculated analytically. It is defined as the integral of the density function

$$
F(x)=\int f(x) d x
$$

For the further calculations the inverse of the cumulative density function $F^{-1}(x)$ is needed. To support a realistic analysis, the density function of the battery cell capacities is not assumed exactly Gaussian but cut at each side at $k \sigma$ and rescaled to fulfill the equation in (5) and (6):

$$
f^{*}(x)=\frac{f(x)}{1-2 F(\mu-k \sigma)}
$$


This is a necessary adaption to prevent extreme capacity values being considered in the calculation.

With $\mu$ and $\sigma$ being the mean value and the standard deviation of the deployed cell, it is assumed that the capacity range of all cells is within $\mu \pm k \sigma$.

The integral of the density function is hence defined as

$$
\int_{-\infty}^{+\infty} f(x) d x=\int_{-\infty}^{+\infty} f^{*}(x) d x=\int_{\mu-k \sigma}^{\mu+k \sigma} f^{*}(x) d x=1 .
$$

The two density functions $f(x)$ and $f^{*}(x)$ are shown in Fig. 4 (for $\mu=10 \mathrm{Ah}, \sigma=0.15 \mathrm{Ah}$ and $k=2$ ).

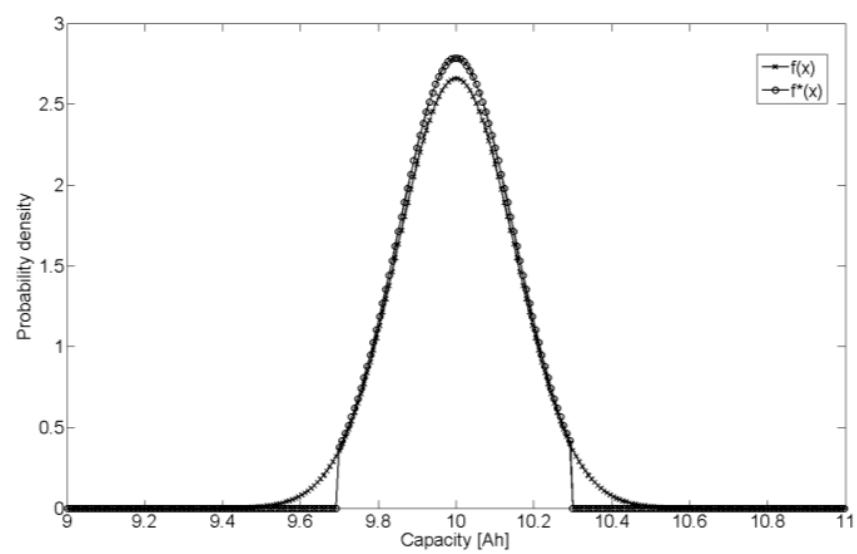

Fig. 4. Gaussian and adjusted density functions $\mathrm{f}(\mathrm{x})$ and $\mathrm{f}^{*}(\mathrm{x})$

\section{B. nS1P Battery systems}

First the variable $k$ is calculated as a function of the number of cells $n$. The density function margins $\mu \pm k \sigma$ are defined such that the probability for a cell having a capacity value between $-\infty$ and $\mu$ - $k \sigma$ is $1 / n$, hence

$$
k=\frac{\mu-F^{-1}\left(\frac{1}{n}\right)}{\sigma} .
$$

During passive balancing, charge is dissipated from cells that are close to their maximum charging voltage. This process lasts until the strongest cell has reached its maximum charging voltage. For a battery system with an $n \mathrm{~S} 1 \mathrm{P}$ configuration $(n$ cells in series, 1 cell in parallel), the charge difference $\Delta Q$ of a cell with a capacity of $x \mathrm{Ah}$ in relation the strongest cell is given as:

$$
\Delta Q=\mu+k \sigma-x
$$

The total balanced charge of all cells can be calculated as follows:

$$
Q_{\text {Bal_pack }}=n \int_{\mu-k \sigma}^{\mu+k \sigma}(\mu+k \sigma-x) f^{*}(x) d x .
$$

Using (3) and (6), (9) can be simplified to

$$
Q_{\text {Bal_pack }}=k n \sigma \text {. }
$$

The energy losses during charging due to passive balancing $W_{\text {Passive }}$ for an $n \mathrm{~S} 1 \mathrm{P}$ battery system sum up to

$$
W_{\text {Passive }}=U_{\text {Bal_avg }} Q_{\text {Bal_pack }}=k n \sigma U_{\text {Bal_avg }} .
$$

$U_{\text {Bal_avg }}$ is the average voltage of the affected cell during the balancing process. For lithium-ion cells, in most applications

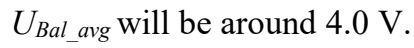

\section{C. $\quad n S m P$ Battery systems}

For battery systems with more than one cell on each stack level $(n \mathrm{~S} m \mathrm{P})$, the calculation has to be adjusted.

Acc. to probability theory basics (see [10]) the resulting mean and standard deviation for independent random variables are $\mu^{\prime}=m \mu$ and $\sigma^{\prime}=\sqrt{m} \sigma$. Using $\mu^{\prime}$ and $\sigma^{\prime}$ instead of $\mu$ and $\sigma$ in (2), the density function becomes

$$
f_{m}(x)=\frac{1}{\sqrt{2 \pi{\sigma^{\prime}}^{2}}} e^{\frac{\left(x-\mu^{\prime}\right)^{2}}{2 \sigma^{\prime 2}}}
$$

In line with (7), the total balanced charge can be written as

$$
Q_{m_{\text {Bal_pack }}}=n \int_{\mu \prime-k \sigma^{\prime}}^{\mu \prime+k \sigma^{\prime}}\left(\mu^{\prime}+k \sigma^{\prime}-x\right) f_{m}^{*}(x) d x .
$$

Using (3) and (6), (13) can be simplified to

$$
Q_{m_{\text {Bal_pack }}}=k n \sigma^{\prime}=k n \sqrt{m} \sigma \text {. }
$$

The energy losses during charging due to passive balancing $W_{m_{\text {Passive }}}$ for an $n \mathrm{~S} m \mathrm{P}$ battery system sum up to

$$
W_{m_{\text {Passive }}}=U_{\text {Bal_avg }} Q_{m_{\text {Bal_pack }}}=k n \sqrt{m} \sigma U_{B a l_{\text {avg }}} \text {. }
$$

\section{CALCULATION OF ACTIVE CHARGE BALANCING PERFORMANCE}

\section{A. Energy savings}

If active balancing is used instead of passive balancing, a considerable share of the equalizing losses is avoided. An overall charge transfer efficiency of around $80 \%$ can be derived from active balancing prototype measurement data (see [11], [12] and [13]) and will be used for numeric calculations below. Using the energy efficiency variable $\eta$, it follows that the energy losses during charging due to active balancing are

$$
W_{m_{\text {Active }}}=(1-\eta) k n \sqrt{m} \sigma U_{B a l_{\text {avg }}}
$$


The balancing effort of a battery system increases with its lifetime as the cell capacities drift apart. Varying operation conditions lead to inhomogeneous aging of individual cells. While the capacity mean value decreases, the standard deviation of the cells increases.

\section{B. Capacity gain}

The following equations refer to $n \mathrm{~S} m \mathrm{P}$ battery packs. For $n \mathrm{~S} 1 \mathrm{P}$ configuration, $m$ can be set to 1 .

The lifetime of a battery pack is usually defined as a minimum remaining capacity in relation to the initial capacity (between 70 and 80 percent for most applications). The weakest cell - or cell level in case of more than one parallel cell - in a battery system determines the effective capacity $C_{\text {Pack }}$ which is in our case

$$
C_{P a c k}=\mu^{\prime}-k \sigma^{\prime}=m \mu-k \sqrt{m} \sigma .
$$

If active balancing is performed during discharge as well, the useable capacity can be increased. For an actively balanced battery pack this capacity value $C_{\text {Pack_active }}$ depends on the efficiency and power of the balancing electronics and can get as high as

$$
C_{\text {Pack_active }}=\mu^{\prime}-(1-\eta) k \sigma^{\prime}=m \mu-(1-\eta) k \sqrt{m} \sigma .
$$

Fig. 5 and Fig. 6 show the effective capacity of a passive respectively active balancing system in terms of cell properties and system configurations.

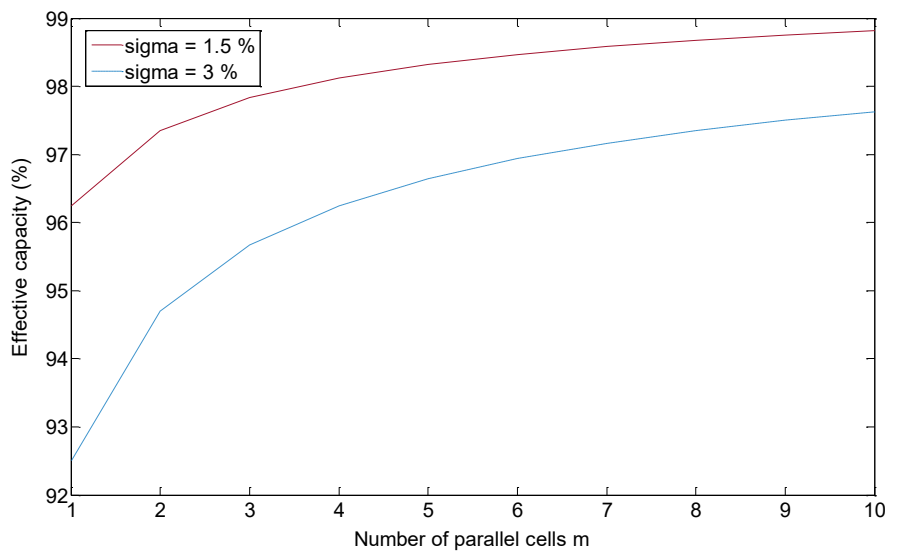

Fig. 5. Effective capacity of a passive balancing battery system over the number of parallel cells acc. to (18) (for $\sigma=1.5 \% / 3 \%$ and $k=2.5$ )

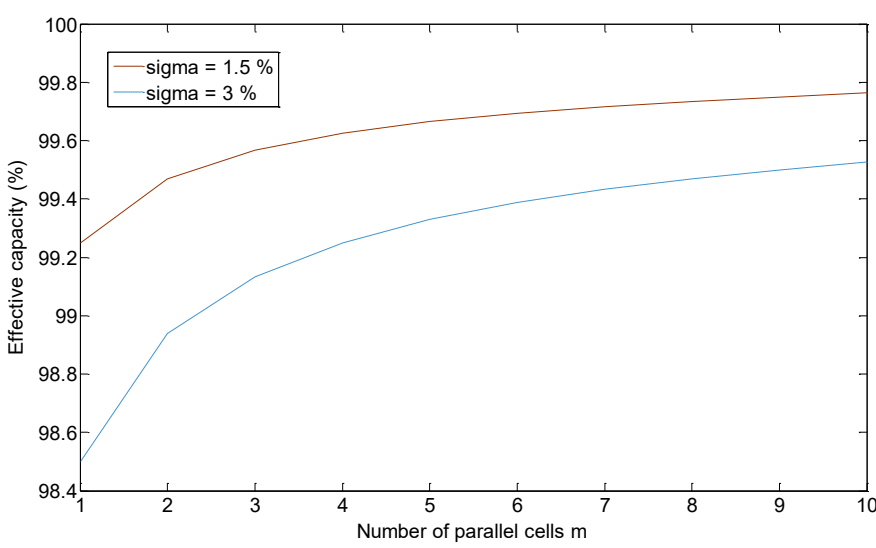

Fig. 6. Effective capacity of an active balancing battery system over the number of parallel cells acc. to (19) (for $\sigma=1.5 \% / 3 \%$ and $k=2.5$ )

\section{NUMERIC SIMULATION}

\section{A. Simulation settings}

The numeric simulations were done using MATLAB. The cell capacity values are randomly generated as a function of the chosen cell parameters and stored in an array. These values are processed acc. to the balancing algorithm shown in Fig. 7: First, the difference of each cell related to an estimated resulting mean value is calculated, based on the balancing efficiency and effort. Then the balancing algorithm decides whether to charge or discharge the cell. After one run, the standard deviation of all cells is computed. This sequence repeats until the deviation reaches less than $0.2 \sigma_{0}$. After 1000 simulations of each parameter set, the mean value of all individual simulation results is computed.

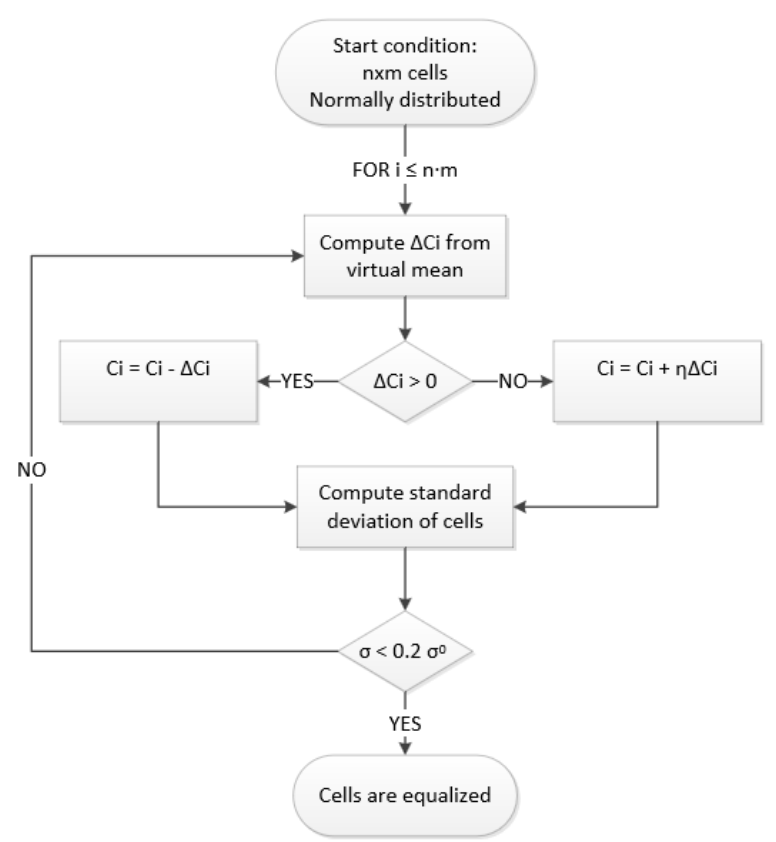

Fig. 7. Algorithm for the cell-to-cell balancing simulation 


\section{A. Simulation results}

Fig. 8 and Fig. 9 show the results of the simulation in comparison with the calculated values. In Fig. 8, the number of parallel cells is varied from 1 to 10 whereas in Fig. 9, the standard deviation of the cell capacities is varied from $1 \%$ to $3 \%$. The overall deviation between simulation and calculation is $0.13 \%$ for Fig. 8 and $0.31 \%$ for Fig. 9. It is explained as a consequence of the iteration steps of the balancing algorithm. The calculated values represent the maximum achievable capacity gain.

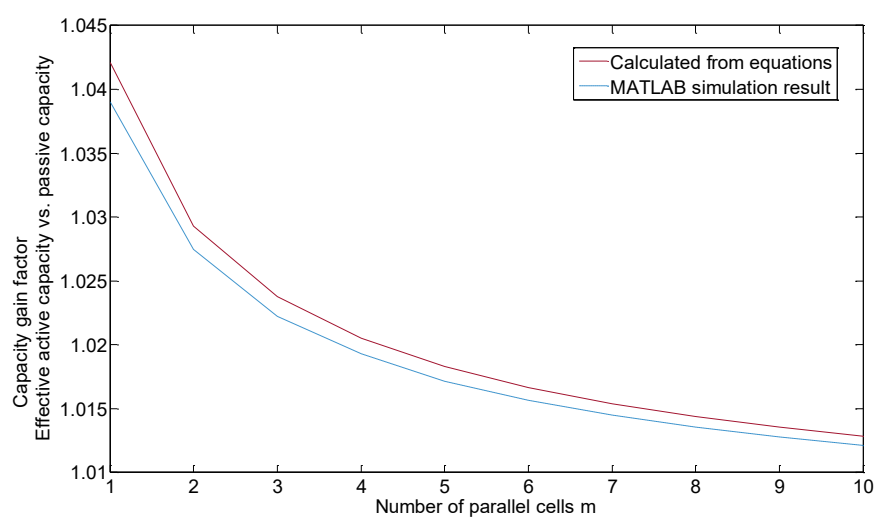

Fig. 8. Capacity gain factor of active and passive balancing as a function of the number of parallel cells acc. to (18) and (19) (for $\sigma=2 \%$ and $k=2.5$ )

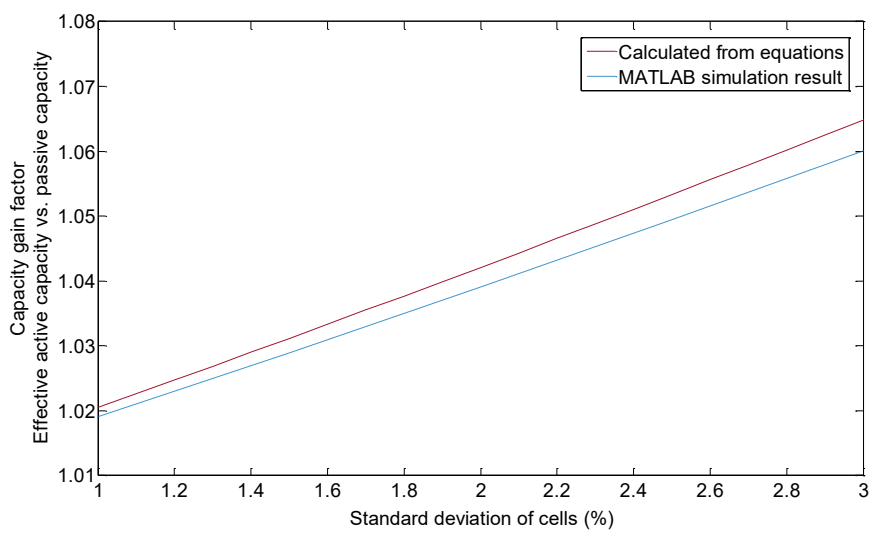

Fig. 9. Capacity gain factor of active and passive balancing as a function of the cell capacity spread acc. to (18) and (19) (for $m=1$ and $k=2.5$ )

\section{CONCLUSION}

It has been shown that the theoretical advantages of active charge balancing in battery systems can be calculated analytically with knowledge of just few cell parameters and the energy efficiency of the active balancing electronics. The achievable capacity gain is a direct consequence of the differences in effective battery capacity for passive and active balancing systems. The behaviour of a cell-to-cell balancing circuit has been simulated in MATLAB for the given battery system settings. The simulation results support the correctness of the derived equations.

The presented results are applicable for balancing circuits based on the cell-to-cell topology, which has been selected for its superior performance in terms of balancing speed and efficiency. An adaption to other topologies mentioned in section I will be a future addition to the present analysis. Due to the fact that the capacity spread of lithium cells increases over lifetime, active charge balancing has the biggest impact in combination with used and nonuniformly aged cells.

As a result of the obtained equations, several options to improve the energy efficiency of passively balanced battery systems can be derived:

- Equations (11) and (15) show that the balancing losses are reduced when more than one cell per level is used in a battery system $(n \mathrm{~S} m \mathrm{P}$ instead of $n \mathrm{~S} 1 \mathrm{P})$.

- Presorted cells have a lower $\sigma$ and lead to fewer balancing losses according to (11) and (15).

- Regarding (11), losses can be reduced if the balancing action is performed not only at the end but rather during the charging process at a lower average cell voltage.

- Uniform operating conditions for all cells shall be ensured to prevent excessive capacity spread over working cycles and lifetime.

Active balancing represents a promising possibility to enhance the energy efficiency and eco friendliness of lithiumion battery systems when used both during the charging and discharging process. Further investigations should be made for battery systems that are not operated in full cycles. Furthermore, cell production is not the only reason for cell capacity variances. Temperature differences that occur during battery operation affect the capacity distribution as well as the aging behavior and shall be considered in future work.

\section{References}

J. Gallardo-lozano, E. Romero-cadaval, M. I. Milanes-montero, and M. A. Guerrero-martinez, "Battery equalization active methods," Journal of Power Sources, vol. 246, pp. 934-949, 2014.

[2] P. Weicker, A Systems Approach to Lithium-Ion Battery Management. Norwood, 2014.

[3] F. Baronti, R. Roncella, and R. Saletti, "Performance comparison of active balancing techniques for lithium-ion batteries," Journal of Power Sources, vol. 267, pp. 603-609, Dec. 2014.

[4] J. Marcinkowski, "Large Li-ion battery packs: Active balancing improves many parameters," EE Times Europe, pp. 1-3, 2014

[5] D. D. Quinn and T. T. Hartley, "Design of novel charge balancing networks in battery packs," Journal of Power Sources, vol. 240, pp. 26-32, Oct. 2013

[6] M. Kauer, S. Narayanaswamy, S. Steinhorst, M. Lukasiewycz, and S. Chakraborty, "Many-to-Many Active Cell Balancing Strategy Design," pp. 267-272, 2015. 
[7] J. Xu, S. Li, C. Mi, Z. Chen, and B. Cao, "SOC Based Battery Cell Balancing with a Novel Topology and Reduced Component Count," Energies, vol. 6, no. 6, pp. 2726-2740, May 2013.

[8] V. Muenzel, J. de Hoog, M. Brazil, D. a. Thomas, and I. Mareels, "Battery Management using Secondary Loads: A Novel Integrated Approach," in IFAC Proceedings Volumes, 2014, vol. 47, no. 3, pp 3924-3929.

[9] S. Paul, C. Diegelmann, H. Kabza, and W. Tillmetz, "Analysis of ageing inhomogeneities in lithium-ion battery systems," Journal of Power Sources, vol. 239, pp. 642-650, Oct. 2013.

[10] L. Meier, Statistik und Wahrscheinlichkeitsrechnung. ETH Zürich, 2015
[11] A. Ramirez and B. Miesch, "BSc Thesis (ET12b): Active balancing Battery Management System for up to 8 Li-Ion cells," ZHAW Zurich University of Applied Sciences, 2015.

[12] M. Einhorn, W. Guertlschmid, T. Blochberger, R. Kumpusch, R. Permann, F. V. Conte, C. Kral, S. Member, and J. Fleig, "A Current Equalization Method for Serially Connected Battery Cells Using a Single Power Converter for Each Cell," vol. 60, no. 9, pp. 42274237, 2011.

[13] F. Baronti, G. Fantechi, S. Member, R. Roncella, and R. Saletti, "High-Efficiency Digitally Controlled Charge Equalizer for SeriesConnected Cells Based on Switching Converter and SuperCapacitor," vol. 9, no. 2, pp. 1139-1147, 2013. 